\title{
Type 1 Diabetes in Athletes
}

\author{
Jane Yardley
}

Alberta Diabetes Institute, Edmonton, Alberta, Canada

DOI: https://doi.org/10.17925/USE.2017.13.02.51

$\mathrm{M}$ anaging training and competition with type 1 diabetes is complicated, but not impossible. Athletes, and their diabetes care providers, should be aware of everything that might impact blood glucose responses to exercise. These factors include the type, timing, intensity and duration of exercise, as well as the timing and size of insulin dosage adjustments and carbohydrate intake. Due to differences in physiology (age, sex, fitness levels, etc.) there is no "one size fits all" solution, however, with careful planning and record-keeping, athletes with type 1 diabetes have the same opportunities for success in their sport as everyone else.

\section{Keywords}

Type 1 diabetes, exercise, insulin, blood glucose, carbohydrate

Disclosure: Jane Yardley has received speakers' fees from Animas Canada and her research has been supported by Medtronic Canada, Ascencia Canada, Animas Canada, Abbott Nutrition Canada, and Lifescan Canada. This article is a short opinion piece and has not been submitted to external peer reviewers. No funding was received in the publication of this article.

Authorship: All named authors meet the International Committee of Medical Journal Editors (ICMJE) criteria for authorship of this manuscript, take responsibility for the integrity of the work as a whole, and have given final approval to the version to be published.

open Access: This article is published under the Creative Commons Attribution Noncommercial License, which permits any noncommercial use, distribution, adaptation, and reproduction provided the original author(s) and source are given appropriate credit.

Received: July 19, 2017

Piblished Online: November 17, 2017

Citation: US Endocrinology, 2017;13(2):51-2

Corresponding Author: Jane Yardley, Alberta Diabetes Institute, 1-002 Li Ka Shing Centre for Health Research Innovation, University of Alberta, Edmonton, AB T6G 2E1, Canada. E: jeyardle@ualberta.ca
Exercise is essential to health and well-being. For people with type 1 diabetes (T1D), it also improves longevity, and decreases the risk of diabetes-related complications. Unfortunately, due to the use of exogenous insulin that stays in circulation substantially longer than endogenous insulin, exercise is also associated with an increased risk of hypoglycemia. This is a major challenge for athletes with T1D who are often training on a daily basis. The level of hypoglycemia risk depends on the type, intensity, timing and duration of training/competition, along with how insulin and carbohydrate intake are managed before and after activity. Other than this, athletes with T1D can train, perform and compete at the same level as those without T1D, provided that blood glucose levels are kept in an appropriate range.

\section{Type and intensity of exercise}

Aerobic activities involve the repeated contraction of large muscle groups. This includes walking, jogging, cycling, swimming, etc., where intensity is low to moderate and the individual performing the activity can maintain a conversation. The risk of hypoglycemia is high with aerobic exercise in people with T1D, but can be diminished by decreasing the amount of insulin in circulation during exercise. This can be achieved by decreasing the long-acting (basal) insulin injection, up to 20\%, the morning of, or the night before, a training sessions for those using multiple daily injections (MDIs); or decrease the basal rate by $50-80 \% 90$ minutes before training, for those using insulin pumps. A further option is to lower the meal/snack bolus by $25-75 \%$ in the hours before training. The decrease in circulating insulin is designed to mimic the body's normal exercise response: insulin secretion decreases while glucagon secretion increases. Target blood glucose levels for starting aerobic exercise should be around 7-10 mmol/L (182-270 mg/dL). There is often a temptation to start aerobic training sessions with blood glucose levels above $10 \mathrm{mmol} / \mathrm{L}$, however, this can hinder performance while also increasing the risk of ketone production and dehydration. Frequent capillary glucose checks are essential, and it is advised to have fast-acting glucose snacks readily available.

At the opposite end of the exercise spectrum, anaerobic activities are short and intense (weight lifting, sprints, intermittent high intensity intervals). Insulin adjustments can be more modest for anaerobic activities, due to the involvement of epinephrine (adrenaline), which causes the liver to release large quantities of glucose to fuel the activity. Consequently, blood glucose levels often increase during anaerobic activities. Emotionally charged/psychologically intense games and competitions can cause a similar response. In addition, muscle glycogen is used as a fuel source, thereby sparing some of the glucose circulating in the blood. Additional insulin may be necessary to compensate for hyperglycemia that may be experienced during anaerobic exercise, or for several hours afterwards. Target blood glucose levels for starting anaerobic activities can be slightly lower (5-7 mmol/L or 90-126 mg/dL), as levels tend to increase, rather than decrease. It should be 
noted, however, that delayed hypoglycemia can be a risk with anaerobic activities, as the body replenishes liver and muscle glycogen stores for 12-24 hours post-activity. This can lead to difficulties with nocturnal hypoglycemia, which can be dangerous, if not fatal. Decreasing basal insulin overnight post-exercise, and consuming a low glycemic index bedtime snack, will help decrease these risks.

\section{Timing of exercise}

Early morning may be the safest training time for T1D athletes, as it is associated with lower circulating insulin levels, due to the time elapsed since the last meal and bolus insulin dose. There are also higher levels of circulating growth hormone and cortisol. This leads to a lower reliance on blood glucose as an energy source, and a greater reliance on lipids. In addition, the body will have up to three meals food intake to replenish glycogen stores before bedtime. Thus, the risk of both immediate and delayed hypoglycemia is generally lower with morning (compared to afternoon) training sessions.

\section{Duration}

Where training sessions are relatively short (i.e., <60-90 minutes), appropriate insulin dose reductions prior to activity may be sufficient to maintain blood glucose levels. Where activities are longer than 60-90 minutes, carbohydrate intake is essential to maintaining a safe range of blood glucose. For durations up to 150 minutes, 30-60 grams/hour of carbohydrate intake is often sufficient to prevent hypoglycemia. Where activity exceeds 150 minutes, 60-90 grams/hour of carbohydrate is recommended. If insulin dosage has not been appropriately reduced before exercise, carbohydrate supplementation must start sooner, with greater hourly amounts being required. It should also be noted that if the athlete has experienced hypoglycemia within the 24 hours before training, that additional carbohydrates are likely to be necessary.

\section{Other considerations}

Athletes with T1D can benefit greatly from continuous glucose monitoring or flash glucose monitoring systems, as these both enable quick and convenient checking of blood glucose levels during and after training and competition. Continuous glucose monitors and sensor augmented insulin pumps have the added advantage of showing blood glucose trends. They also provide alarms when blood glucose levels drop rapidly, or have reached a level where taking action is required. This will help T1D athletes train and compete with more confidence. It may even be beneficial for weight loss and/or maintenance, as it can decrease the amount of carbohydrate that is taken unnecessarily due to fear of hypoglycemia.

Individual responses to exercise vary greatly from patient to patient. Age, sex, and physical fitness all affect blood glucose responses to exercise, as does the overall metabolism of the athlete. In trying to find the appropriate insulin adjustments and carbohydrate intake for different types and durations of training/competition, T1D athletes should be encouraged to keep a detailed diary of everything that can affect blood glucose outcomes. These factors include the type, timing, intensity, and duration of the training session/competition, pre-exercise insulin and food intake, menstrual cycle phase for females, along with overall health, mood, and environmental conditions. Adjusting one variable at a time will help find the "sweet spot" for all of these factors and maximize both safety and performance for the athlete. $\square$

\section{Recommended reading}

1. Riddell MC, Gallen IW, Smart CE et al Exercise management in type 1 diabetes: a consensus statement, Lancet Diabetes Endocrinol, 2017.5.377-90.

2. Colberg SR, Sigal RJ, Yardley JE, et al., Physical activity/exercise and diabetes : a position statement of the American diabetes association, Diabetes Care, 2016;39:2065-79.

3. Yardley JE, Colberg SR, Update on the management of type 1 diabetes and type 2 diabetes in athletes, Curr Sports Med Rep, 2017;16:38-44.

. Gallen I, Type 1 Diabetes: clinical management of the athlete, London: Springer Verlag, 2012. 